\title{
Systematics and uncertainties of Hamiltonian parameters of soft-rotator model for actinides and their implications for nuclear data calculations
}

\author{
S. Chiba ${ }^{1, a}$, H. Koura ${ }^{1}$, T. Maruyama ${ }^{1}$, S. Kunieda ${ }^{1}$, and E. Sh. Soukhovitskiî̃ ${ }^{2}$ \\ 1 Japan Atomic Energy Agency, Tokai, Naka, Ibaraki 319-1195, Japan \\ 2 Joint Institute for Energy and Nuclear Research, 220109 Minsk-Sosny, Beralus
}

\begin{abstract}
The collective low-lying levels of even-even actinides are analyzed in terms of the soft-rotator model (SRM), and best fit parameters of SRM Hamiltonian are deduced for 19 nuclides. Systematics of these parameters are then obtained as a function of the ground-state quadrupole deformation. It was found that there is a possibility of extending such a systematics to include the fission product nuclei. Furthermore, we propose a method to estimate the covariance of the SRM parameters and its impact on the cross sections calculated with them quantitatively. It is shown that low-energy observables are more sensitive to the uncertainties in the SRM parameters, therefore, sensitive to the collective structure effects.
\end{abstract}

\section{Introduction}

Excitation of low-lying collective levels is an important characteristics of nucleon-induced nuclear reactions for low- to medium-energy range directly relevant to nuclear data evaluation. It is well known that the concise calculation like spherical optical model fails to reproduce measured data for heavy fission-product and actinide regions since they are highly deformed nuclei. Furthermore, many of the spherical nuclei exhibit collective vibrational excitation. Therefore it is essential to take these collective effects into account into nuclear data calculation accurately.

We have been developing and applying a computational scheme based on the soft-rotator model (SRM) and a coupledchannel method based on it (SRM-CC) for many years[1-7]. In this approach, we start considering the nature of lowlying collective levels of even-even nuclei, assigning specific quantum numbers of rotational and vibrational motions, and we search SRM Hamiltonian parameters to reproduce the level energies. These parameters are then used to calculate their coupling strengths, like the factor $\left\langle G . S .\left|\beta_{2}^{n}\right| J_{q}^{\pi}\right\rangle$, in the coupledchannel calculation. The SRM parameters appear through the description of the SRM wave functions, e.g., $|G . S$.$\rangle and$ $\left|J_{q}^{\pi}\right\rangle$, where $q$ denotes a set of quantum numbers of SRM. We have seen that the coupling strengths calculated by SRM are noticeably different from those by simple rigid rotor or harmonic vibrational model in many cases. This SRM-CC approach has turned out to be a powerful tool for calculation of nuclear data for low- to medium-energy range.

Normally, the SRM Hamiltonian parameters are determined for each specific nucleus. However, for some nuclei, only limited information is available. In such cases, we cannot determine the SRM Hamiltonian parameters, and consequently, we cannot get a good estimation of the cross section data.

In the present work, we apply the SRM to some eveneven actinides, and deduce the SRM Hamiltonian parameters for each nucleus. Then, we try to find a systematical trend

\footnotetext{
${ }^{a}$ Presenting author, e-mail: chiba.satoshi@jaea.go.jp
}

from the results, which will be used for nuclei for which the collective level information is missing or incomplete. Furthermore, we propose a method to estimate covariance of the SRM parameters and that of the cross sections calculated with them. This method will be applied to ${ }^{238} \mathrm{U}$ and ${ }^{250} \mathrm{Cm}$ to demonstrate the magnitude of the uncertainties brought by SRM parameters as typical examples.

\section{SRM analysis of low-lying collective levels of even-even actinides}

The SRM analysis was carried out for the 19 even-even actinide nuclei listed in table 1 . In this table, the ground-state quadrupole deformation parameter $\beta_{20}$ and number of levels considered for each collective band are listed. In the present work, the ground-state rotational band (G.S. rot.), $\mathrm{K} \approx 2$ band, $\beta_{2}$-vibrational band $\left(n_{\beta_{2}}=1\right)$, negative parity octupole band $\left(\pi_{\beta_{3}}=-1\right)$ and $\gamma$-vibrational band $\left(n_{\gamma}=1\right)$ are considered. The meaning of the variables and symbols are explained in ref. [8]. The experimental levels are taken mostly from the recent version of the Nuclear Data Sheets, while some of recent experimental data are also employed, e.g., ref. [9].

The SRM Hamiltonian parameters are adjusted to reproduce the level energies in table 1 for each nucleus on the basis of $\chi^{2}$ minimization. After these searches are finished, their systematical trend was sought for as a function of $\beta_{20}$, and new initial values are generated. That parameter search was carried out several times until a good convergence was achieved. An example of the levels calculated with the best fit parameter is displayed in figure 1 for ${ }^{238} \mathrm{Pu}$, for which levels in the whole bands are experimentally known. We adopted the $\beta_{20}$ value from the work of Koura, Tachibana, Uno and Yamada known as KTUY05 mass model [10]. Then, each parameter was fitted by a simple polynomial of the second order (or less) as a function of $\beta_{20}$. Example of this fitting is shown in figure 2 for the $\hbar \omega_{0}$ parameter. We notice a clear systematical trend. In this figure, the results of nuclei for which levels are known in 5 bands are shown as full circles, while those of other nuclei are plotted with open circles. We put 
Table 1. Nuclei, their ground-state quadrupole deformation parameter $\beta_{20}[10]$, and number of levels in each band adopted in SRM analysis.

\begin{tabular}{ccccccc}
\hline Nucleus & $\beta_{20}$ & G.S. rot. & $K \approx 2$ & $\mathrm{n}_{\beta_{2}}=1$ & $\pi_{\beta_{3}}=-1$ & $\mathrm{n}_{\gamma}=1$ \\
\hline${ }^{224} \mathrm{Th}$ & 0.176 & 6 & & & 5 & \\
${ }^{226} \mathrm{Th}$ & 0.194 & 6 & & 2 & 5 & \\
${ }^{228} \mathrm{Th}$ & 0.203 & 8 & 3 & 2 & 4 & \\
${ }^{230} \mathrm{Th}$ & 0.217 & 6 & 3 & 3 & 4 & \\
${ }^{232} \mathrm{Th}$ & 0.235 & 6 & 4 & 3 & 3 & \\
${ }^{232} \mathrm{U}$ & 0.231 & 6 & 3 & 3 & 2 & \\
${ }^{234} \mathrm{U}$ & 0.242 & 7 & 5 & 3 & 3 & 2 \\
${ }^{236} \mathrm{U}$ & 0.258 & 9 & 4 & & 5 & \\
${ }^{238} \mathrm{U}$ & 0.264 & 6 & 4 & 3 & 2 & 3 \\
${ }^{238} \mathrm{Pu}$ & 0.264 & 7 & 3 & 2 & 3 & 2 \\
${ }^{240} \mathrm{Pu}$ & 0.274 & 6 & 2 & 3 & 3 & \\
${ }^{242} \mathrm{Pu}$ & 0.280 & 7 & 1 & 2 & 3 & \\
${ }^{242} \mathrm{Cm}$ & 0.282 & 4 & & & & \\
${ }^{244} \mathrm{Cm}$ & 0.289 & 5 & & & & \\
${ }^{246} \mathrm{Cm}$ & 0.291 & 5 & 3 & 2 & 3 & 3 \\
${ }^{248} \mathrm{Cm}$ & 0.295 & 8 & 2 & 3 & 3 & \\
${ }^{250} \mathrm{Cm}$ & 0.301 & 7 & & & & \\
${ }^{250} \mathrm{Cf}$ & 0.299 & 4 & 3 & 2 & 2 & 2 \\
${ }^{252} \mathrm{Cf}$ & 0.301 & 3 & 3 & & & \\
\hline
\end{tabular}

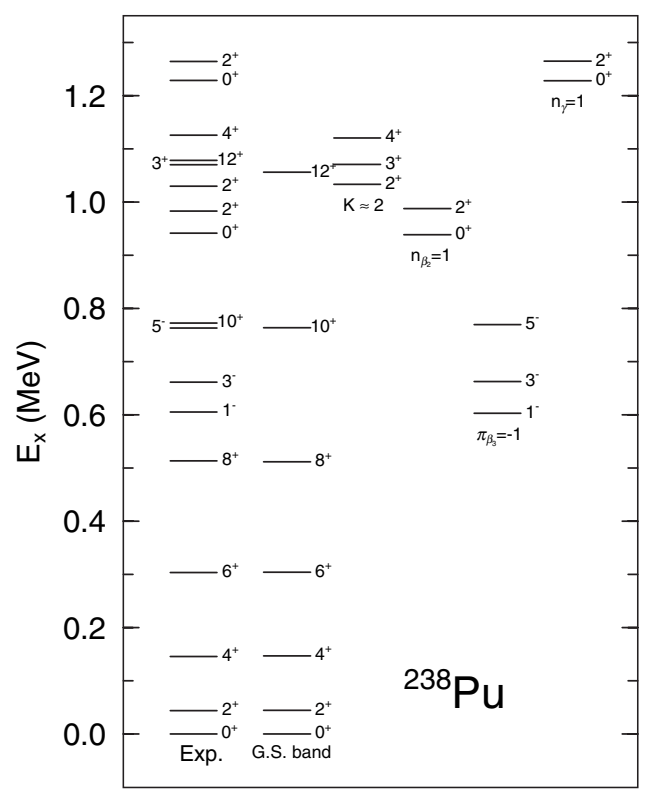

Fig. 1. Low-lying collective levels of ${ }^{238} \mathrm{Pu}$. The experimentally known levels are shown at the leftmost column, while results of the SRM calculation with best-fit parameters are shown to the right for various band levels.

a certain weight on the full circles. The obtained polynomial coefficients are shown in table 2 . Such a systematics can be used to estimate the low-lying collective levels of nuclei for which they are not known or only partially known. We have used the systematics of the parameters obtained and compared the SRM results with experimental data[9] for ${ }^{250} \mathrm{Cm}$ in figure 3 . We notice that not only the experimentally known

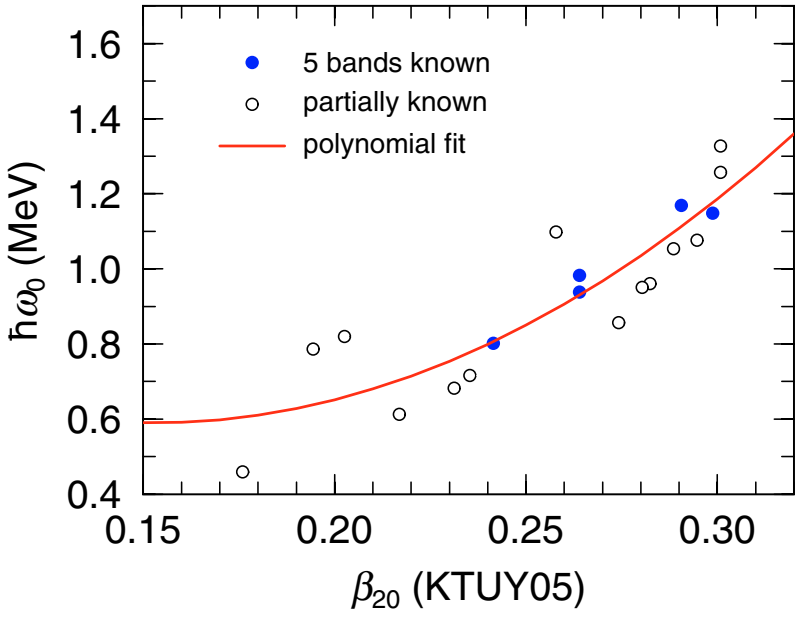

Fig. 2. Obtained $\hbar \omega_{0}$ parameter (symbols) and a polynomial fitting to it (smooth curve).

Table 2. Polynomial coefficients to reproduce the systematical trend of SRM parameters obtained from the global analysis of low-lying collective levels of actinides. The parameters are expressed as $P=$ $a \times \beta_{20}^{2}+b \times \beta_{20}+c$, where $\beta_{20}$ denotes the ground-state quadrupole deformation parameter deduced from KTUY05 mass model[10]. These parameters must be used in the range of the present analysis, namely, $0.17 \leq \beta_{20} \leq 0.3$.

\begin{tabular}{crrr}
\hline Parameter & $\mathrm{a}$ & $\mathrm{b}$ & $\mathrm{c}$ \\
\hline$\hbar \omega_{0}$ & 27.53 & -8.418 & 1.233 \\
$\mu_{\beta_{20}}$ & 6.464 & -4.698 & 1.016 \\
$\mu_{\gamma_{0}}$ & & -0.2813 & 0.3253 \\
$\gamma_{0}$ & & -0.2425 & 0.2193 \\
$a_{42}$ & & & 0.01494 \\
$\gamma_{4}$ & & -0.006834 & 0.07791 \\
$\delta_{4}$ & & -0.001748 & 0.7045 \\
$\epsilon_{0}$ & & -0.05671 & 0.1751 \\
$\eta$ & & -0.01289 & 0.006875 \\
$\mu_{\epsilon}$ & & 0.01708 & 0.4522 \\
$a_{32}$ & & -0.02574 & 0.02591 \\
$\delta_{n}$ & 659.9 & -152.1 & 10.20 \\
\hline
\end{tabular}

G.S. band levels are reproduced fairly well but that other missing levels are predicted above the excitation energy of 1.2 MeV rather densely. These estimated levels will modify the direct inelastic cross sections and as well as the level density, affecting the statistical model calculation considerably.

Furthermore, we have investigated a possibly to get systematics for a wider mass region by combining the present results with an analysis in the fission production region [11]. The result is shown in figure 4 for $\mu_{\beta_{2}}$ parameter. We can find a surprisingly smooth trend ranging from small $\beta_{20}$ region (probably vibrational nuclei) to large deformed nuclei, which include both the actinide and heavy FP nuclei.

\section{Covariance analysis}

Next, we try to calculate the uncertainties of the estimated SRM parameters and their impacts to the cross sections 


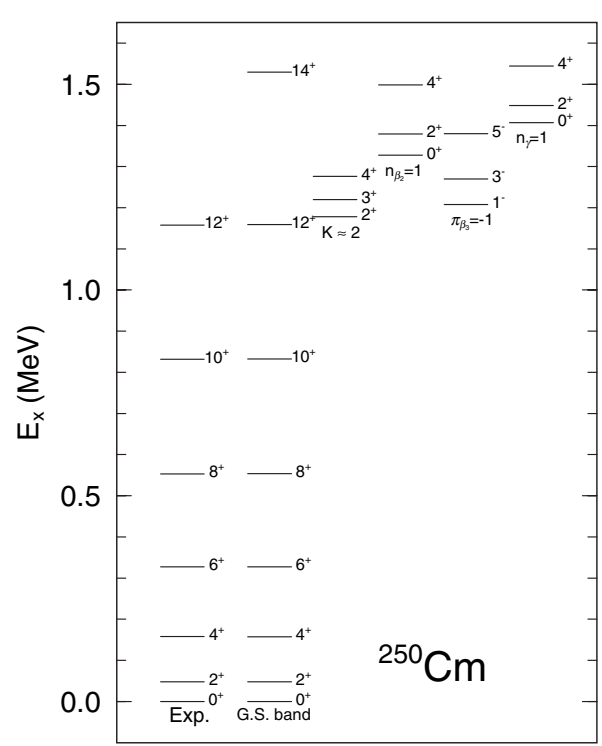

Fig. 3. Low-lying collective levels of ${ }^{250} \mathrm{Cm}$. The experimentally known levels are shown at the leftmost column, while results of the SRM calculation using the systematics obtained are given for various band levels to the right. Some SRM parameters were readjusted to reproduce the observed levels of this nucleus.

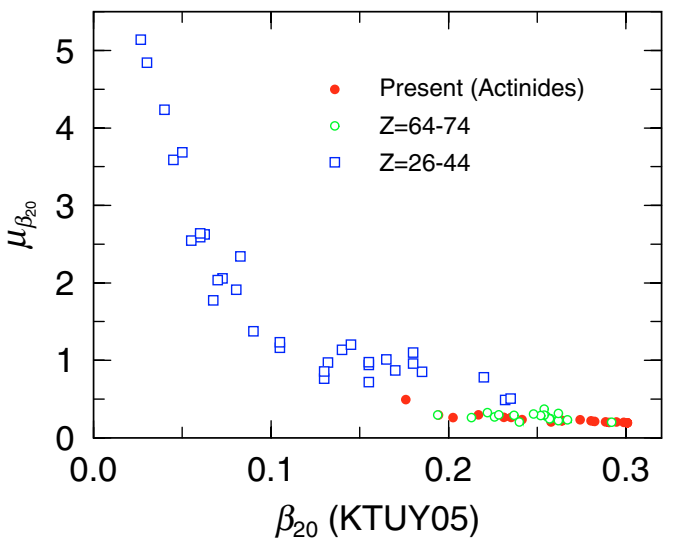

Fig. 4. The $\mu_{\beta_{20}}$ parameter obtained presently for actinides (filled circles) and preliminary for FP nuclei (open circles and squares).

calculated using them. By starting from the covariance $\mathbf{V}$ of the level energy vector $\mathbf{E}$ to which the SRM parameters are adjusted, we use the law of error propagation twice to obtain the covariance $\mathbf{C}$ of the calculated cross sections $\sigma$ :

$$
\begin{aligned}
& \mathbf{C}=\mathbf{H Z \mathbf { H } ^ { \top }}, \text { and } \\
& \mathbf{Z}=\left(\mathbf{G}^{\top} \mathbf{V}^{-1} \mathbf{G}\right)^{-1} .
\end{aligned}
$$

Here, $\mathbf{G}$ and $\mathbf{H}$ are sensitivity matrices given as

$$
\begin{aligned}
& \mathbf{G}=\left(G_{i, j} \mid G_{i, j}=\frac{\partial E_{i}^{\mathrm{cal}}}{\partial P_{j}}\right), \text { and } \\
& \mathbf{H}=\left(H_{i, j} \mid H_{i, j}=\frac{\partial \sigma_{i}}{\partial P_{j}}\right),
\end{aligned}
$$

while $\mathbf{Z}$ is the covariance matrix of SRM parameters represented by a vector $\mathbf{P}\left(=P_{j}, j=1, \ldots\right.$, number of parameters $)$.
Table 3. Names (leftmost column), their uncertainties in \% (second column) and lower-half of the correlation matrix multiplied by 1000 for the "key" SRM parameters used in parameter adjustment for ${ }^{238} \mathrm{U}$.

\begin{tabular}{cccrrrr}
\hline Name & Error (\%) & $\hbar \omega_{0}$ & $\mu_{\beta_{20}}$ & $\mu_{\gamma_{0}}$ & $\gamma_{0}$ & $\delta_{n}$ \\
\hline$\hbar \omega_{0}$ & 0.415 & 1000 & & & & \\
$\mu_{\beta_{20}}$ & 0.254 & -886 & 1000 & & & \\
$\mu_{\gamma_{0}}$ & 0.322 & 27 & 204 & 1000 & & \\
$\gamma_{0}$ & 0.231 & -14 & 220 & 169 & 1000 & \\
$\delta_{n}$ & 0.478 & 6 & -273 & -283 & -262 & 1000 \\
\hline
\end{tabular}

Table 4. Same as table 3 but for ${ }^{250} \mathrm{Cm}$.

\begin{tabular}{ccrrr}
\hline Parameter Name & Error(\%) & $\hbar \omega_{0}$ & $\mu_{\beta_{20}}$ & $\gamma_{0}$ \\
\hline$\hbar \omega_{0}$ & 48.0 & 1000 & & \\
$\mu_{\beta_{20}}$ & 27.3 & -1000 & 1000 & \\
$\gamma_{0}$ & 143 & 997 & -997 & 1000 \\
\hline
\end{tabular}

We assume that the errors of the level energies are diagonal and represented with a constant fractional error $f$ :

$$
\mathbf{V}=\left\{V_{i, j} \mid V_{i, j}=\left(f E_{i}^{\mathrm{exp}}\right)^{2} \delta_{i, j}\right\} .
$$

The constant $f$ was determined with the condition that

$$
f=\sqrt{\frac{1}{N} \sum_{i=1}^{N}\left(\frac{E_{i}^{\mathrm{exp}}-E_{i}^{\mathrm{cal}}}{E_{i}^{\mathrm{exp}}}\right)^{2}},
$$

namely, the $\chi^{2}$ divided by number of level data $N$ is normalized to be unity. Elements of the inverse of $\mathbf{Z}$ are then obtained as

$$
Z_{i, j}^{-1} \equiv\left(G^{T} V^{-1} G\right)_{i, j}=f^{-2} \sum_{k=1}^{N} G_{k, i}\left(E_{k}^{\exp }\right)^{-2} G_{k, j} .
$$

This equation shows that the error of the SRM parameter is directly proportional to the factor $f$, i.e., a good fit leads to small errors of the parameters.

It must be noted that the level energies are determined experimentally to a much higher accuracy than that of theoretical prediction. Therefore, the uncertainties assigned to the diagonal elements of $V$ actually mean the uncertainty of the model itself.

By using this procedure, we calculate the covariance matrix of SRM parameters, and identify which parameters are dominant ("key") to describe the collective level structure. Such an identification is important since some of the SRM parameters have a rather weak sensitivity to the prediction of energy levels while the sensitivity can be significantly larger for cross section calculation. Therefore we need to restrict the number of parameters that are allowed to vary in the calculation of the covariance matrix.

In table 3, the covariance matrix of "key" SRM parameters for ${ }^{238} \mathrm{U}$ is shown. The parameters are obtained to a high accuracy since whole 5 bands are known. Similar results for ${ }^{250} \mathrm{Cm}$ is shown in table 4 , for which only 3 parameters could be identified as "key" due to the fact that only the ground-state band members are known for this nucleus. Furthermore, the uncertainties of the obtained parameters are larger by more 
Table 5. Uncertainty of calculated cross sections for ${ }^{238} \mathrm{U}$ and ${ }^{250} \mathrm{Cm}$ due to uncertainties of SRM parameters in tables 3 and 4 , respectively.

\begin{tabular}{crr}
\hline Quantity & $\begin{array}{r}\text { Error (\%) of } \\
{ }^{238} \text { U c.s. }\end{array}$ & $\begin{array}{r}\text { Error (\%) of } \\
{ }^{250} \text { Cm c.s. }\end{array}$ \\
\hline $\mathrm{S}_{0}$ & 0.18 & 2.6 \\
$\mathrm{~S}_{1}$ & 0.14 & 21.4 \\
$\sigma_{T}(10 \mathrm{keV})$ & 0.067 & 3.6 \\
$\sigma_{T}(1 \mathrm{MeV})$ & 0.079 & 0.52 \\
$\sigma_{T}(2 \mathrm{MeV})$ & 0.061 & 0.087 \\
$\sigma_{R}(10 \mathrm{keV})$ & 0.15 & 0.081 \\
$\sigma_{R}(1 \mathrm{MeV})$ & 0.097 & 1.5 \\
$\sigma_{R}(2 \mathrm{MeV})$ & 0.11 & 1.3 \\
$\sigma_{e l}(10 \mathrm{keV})$ & 0.072 & 6.0 \\
$\sigma_{e l}(1 \mathrm{MeV})$ & 0.13 & 0.68 \\
$\sigma_{e l}(2 \mathrm{MeV})$ & 0.044 & 1.21 \\
$\sigma_{i n}^{2^{+}}(1 \mathrm{MeV})$ & 0.57 & 6.8 \\
$\sigma_{i n}^{2^{+}}(2 \mathrm{MeV})$ & 0.24 & 0.81 \\
$\sigma_{i n}^{T}(1 \mathrm{MeV})$ & 0.55 & 9.6 \\
$\sigma_{i n}^{T}(2 \mathrm{MeV})$ & 0.21 & 1.4 \\
\hline
\end{tabular}

than a factor of 100 compared to those of ${ }^{238} \mathrm{U}$. The influence of uncertainties of the SRM parameters in tables 3 and 4 on the calculated cross sections are shown in table 5. Here, the s- and p-wave strength functions $\left(S_{0}\right.$ and $\left.S_{1}\right)$, the total $\left(\sigma_{T}\right)$, reaction $\left(\sigma_{R}\right)$, elastic scattering cross sections $\left(\sigma_{e l}\right)$ at $10 \mathrm{keV}, 1 \mathrm{MeV}$ and $2 \mathrm{MeV}$, and direct inelastic scattering cross section to the 1 st excited $2^{+}$state $\left(\sigma_{i n}^{2^{+}}\right)$and total direct inelastic scattering cross sections $\left(\sigma_{i n}^{T}\right)$ at $1 \mathrm{MeV}$ and $2 \mathrm{MeV}$ are selected. These quantities are calculated by SRM-CC method with a code OPTMAN[8]. We notice from this table that the uncertainty is larger in general at low energy than higher ones; compare, e.g., the uncertainties in $\sigma_{T}$ of ${ }^{250} \mathrm{Cm}$ at $10 \mathrm{keV}, 1 \mathrm{MeV}$ and $2 \mathrm{MeV}$ and $\sigma_{i n}^{T, 2^{+}}$at 1 and $2 \mathrm{MeV}$. Still, the uncertainties in the calculated cross sections are quite small for ${ }^{238} \mathrm{U}$ because of the good fit of levels obtained with SRM. However, for other nuclei for which we have to use the systematics of SRM parameters, uncertainties in their SRM parameters may be much larger (possibly several hundred times larger). See, e.g., figure 2 and table 4 . For those cases, the uncertainties in the SRM parameters introduce non-negligible contribution to the calculated cross sections, especially at low energies. In case of ${ }^{40} \mathrm{Ar}$ for which SRM is not able to describe the collective levels energies in the same accuracy as ${ }^{238} \mathrm{U}$ [7], uncertainties in some of the cross sections due to those of SRM parameters can be almost $50 \%$.

Normally, effects of these uncertainties are "absorbed" by the adjustment of the optical model (OM) parameters. It means, however, the OM parameters thus obtained are influenced by the inaccuracy of the SRM parameters and shifted from the "adequate" values. The inadequacy of using the spherical OM or simple rotational or vibrational models is evident from this context for nuclei exhibiting large collectivity. This fact may account for the difficulty in explaining, e.g., the p-wave strength function with simple models.

\section{Concluding remarks}

We have analyzed the low-lying collective level structure of 19 even-even actinide nuclei in terms of the soft-rotator model (SRM) and deduced SRM Hamiltonian parameters. Clear systematics was found for some "key" parameters as a functions of ground-state quadrupole deformation parameter. They were expressed as simple polynomials and their coefficients are presented. Such a systematics seems to be extendable to the fission products, and a more universal systematics may be constructed in the near future. Then we proposed a method to estimate the uncertainties of the SRM parameters and some numerical examples are shown. Influence of these uncertainties on the calculated cross sections are demonstrated. When the SRM parameters are determined accurately for nuclei whose low-lying collective levels are well known, the influence can be negligible. For other cases, however, the uncertainties in the cross section can be significant, especially at low energies, and influence the determination of optical model parameters. Such effects are known by experience, but we have given a method to discuss it in a quantitative way in this work. This fact shows the importance of taking into account the collective structure effects accurately in cross section calculation. It should be stressed, however, that the uncertainties in the SRM parameters can be drastically reduced when we can employ the Bayes' theorem, namely, considering cross section data to further determine them, where the covariance of SRM parameters V obtained in this work is used as "a priori" covariance.

The authors would like to thank Dr R. Capote of IAEA for fruitful discussions on use of SRM-CC. A part of this work was carried out under auspices of Grants-in-Aid for Scientific Research (18560805) of JSPS.

\section{References}

1. S. Chiba, O. Iwamoto, Y. Yamanouti, M. Sugimoto, M. Mizumoto, K. Hasegawa, E.Sh. Sukhovitskĩ̃, Y.V. Porodzinskiĩ, Y. Watanabe, Nucl. Phys. A 624, 305 (1997).

2. E.Sh. Sukhovitskiı̃, Y.O. Lee, J. Chang, S. Chiba, O. Iwamoto, Phys. Rev. C 62, 044605 (2000).

3. S. Chiba, O. Iwamoto, E.Sh. Sukhovitsukiñ, Y. Watanabe, T. Fukahori, J. Nucl. Sci. Technol. 37, 498 (2000).

4. E.Sh. Soukhovitskiĩ, S. Chiba, J.-Y. Lee, Y.-O. Lee, J. Chang, T. Maruyama, O. Iwamoto, J. Nucl. Sci. Technol. 39, 816 (2002).

5. E.Sh. Sukhovitskiĩ, S. Chiba, J.-Y. Lee, B.-T. Kim, S.-W. Hong, J. Nucl. Sci. Technol. 40, 69 (2003).

6. W. Sun, Y. Watanabe, E.Sh. Sukhovitskiĩ, O. Iwamoto, S. Chiba, J. Nucl. Sci. Technol. 40, 635 (2003).

7. N.T. Okumuşog̃lu, F. Korkmaz Gorur, J. Birchall, E.Sh. Soukhovitskii, R. Capote, J.M. Quesada, S. Chiba, Phys. Rev. C 75, 034616 (2007).

8. E.Sh. Soukhovitski, S. Chiba, O. Iwamoto, K. Shibata, T. Fukahori, G.B. Morogovskij, Programs OPTMAN and SHEMMAN, version 8 (2004), JAERI-Data/Code 2005-002 (2005).

9. T. Ishii et al., J. Phys. Soc. Japan 75, 043201 (2006).

10. H. Koura, T. Tachibana, M. Uno, M. Yamada, Prog. Theor. Phys. 113, 305 (2005).

11. S. Kunieda, S. Chiba et al. (these proceedings). 